\title{
Measurement of the transient shielding effectiveness of shielding cabinets
}

\author{
H. Herlemann and M. Koch
}

University of Hannover, Institute of Electrical Engineering and Measurement Science, Hannover, Germany

\begin{abstract}
Recently, new definitions of shielding effectiveness (SE) for high-frequency and transient electromagnetic fields were introduced by Klinkenbusch (2005). Analytical results were shown for closed as well as for non closed cylindrical shields. In the present work, the shielding performance of different shielding cabinets is investigated by means of numerical simulations and measurements inside a fully anechoic chamber and a GTEM-cell. For the GTEMcell-measurements, a downscaled model of the shielding cabinet is used. For the simulations, the numerical tools CONCEPT II and COMSOL MULTIPHYSICS were available. The numerical results agree well with the measurements. They can be used to interpret the behaviour of the shielding effectiveness of enclosures as function of frequency. From the measurement of the electric and magnetic fields with and without the enclosure in place, the electric and magnetic shielding effectiveness as well as the transient shielding effectiveness of the enclosure are calculated. The transient SE of four different shielding cabinets is determined and discussed.
\end{abstract}

\section{Introduction}

The question of how to determine the protective properties of a shield against electromagnetic disturbances is a difficult one to be addressed, even in frequency domain. According to conventional standard measurement procedures (IEEE Standard 299-1997), only the electric- or magnetic SE of the empty shield is measured. Since the shield usually forms a cavity resonator with internal resonances, the shielding effectiveness breaks down at the cavity's characteristic resonance frequencies. In addition, it becomes a function of po-

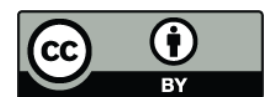

Correspondence to: H. Herlemann (herlemann@ieee.org) sition. The effects on the behaviour of the SE when a filling (e.g. a printed circuit board, PCB, inside a small enclosure) is present are even less predictable since on the one hand part of the electromagnetic energy is absorbed and on the other hand, there are numerous coupling paths into the electronic equipment. As was shown by Marvin et al. (2004, 2005), the reduction in absorbed power inside the filling when the shield is applied is less over most of the frequencies, than the SE value indicates. It was found out, that the conventional SE value of the empty shield exhibits an overestimate concerning the absorbed power at all but the resonance frequencies. The authors suggested a test setup where the shield is loaded with a replacement content that represents the original content (i.e. PCB) by having same dimensions and electrical material parameters. A different approach to determine the protection properties of a loaded shield was introduced by Herlemann et al. 2004. Concerning the susceptibility of electronic equipment against transient electromagnetic fields, the authors suggested to determine the decrease of the breakdown probability of electronic equipment when the shield is applied. It is a statistical approach.

Whereas the mentioned authors introduced measurement procedures with loaded enclosures, Klinkenbusch (2005) suggested measurements of the empty shield from which the effect of the shield on the specific absorption rate (SAR) of the filling is calculated. The two analytically developed measures introduced by Klinkenbusch are namely the electromagnetic shielding effectiveness (EM-SE) in the frequency domain and the transient shielding effectiveness (T-SE) in the time domain. EM-SE is a function of frequency and T-SE is a function of the specific field pulse against which the shield's protective property is examined (e.g. electrostatic discharge, ESD, high power microwave, HPM, ultra wide band (UWB) pulses).

In this contribution, the shielding behaviour of shielding cabinets is examined. The enclosure under test is a $3 \times 3 \times 2.5 \mathrm{~m}$ metallic cabin introduced by Frenzel (2007).

Published by Copernicus Publications on behalf of the URSI Landesausschuss in der Bundesrepublik Deutschland e.V. 


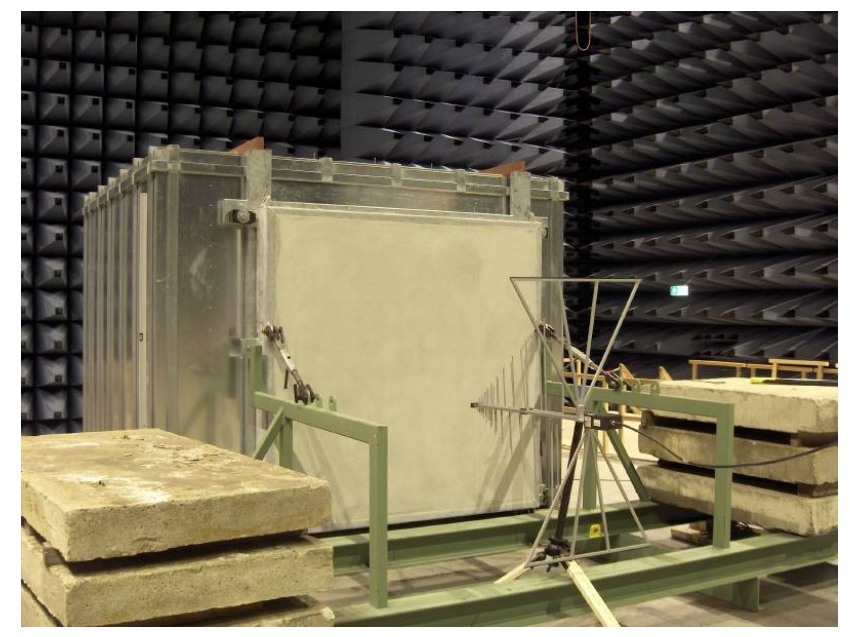

Fig. 1. Shielding cabinet inside a fully anechoic chamber (Frenzel et al., 2007).

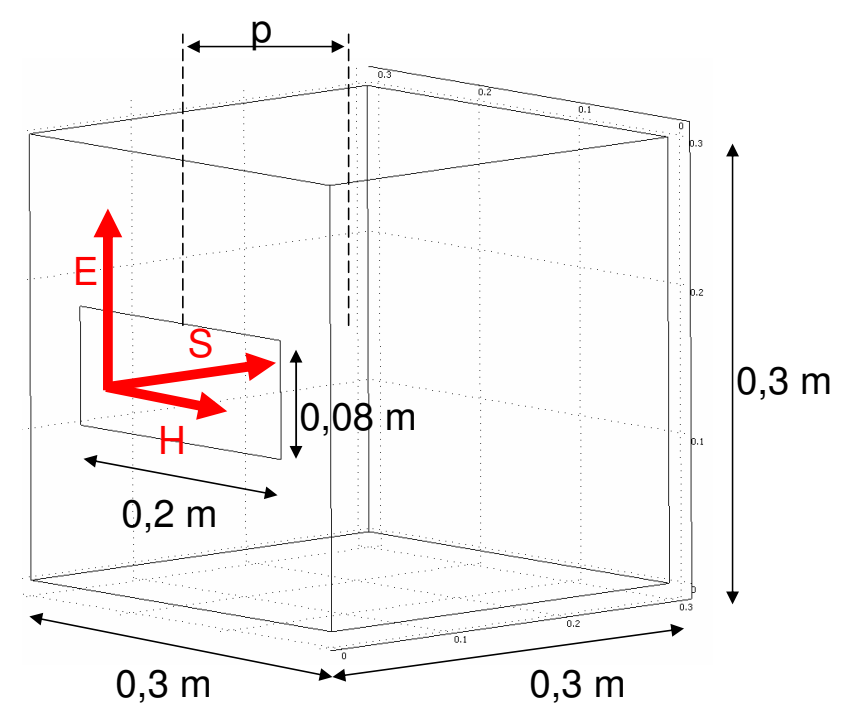

Fig. 2. Scaled model of the shielding cabinet for numerical simulations and shielding SE inside a GTEM-cell.

One side wall contains a $1.9 \times 1.9 \mathrm{~m}$ squared opening in front of which different shielding walls can be mounted (see Fig. 1). By means of exchanging this shielding wall, a new measurement configuration is maintained. Thus, different shielding cabinets can be examined. In order to be able to interpret results of SE measurements, a scaled model (scaling factor 1:10) of the shielding cabinet is examined numerically and by means of measurements inside a GTEM-cell. For the numerical examinations, the Method of Moment based tool CONCEPT II and the Finite Element Method based tool COMSOL MULTIPHYSICS is used. Measurements of the T-SE of the shielding cabinet are performed inside a large fully anechoic chamber.

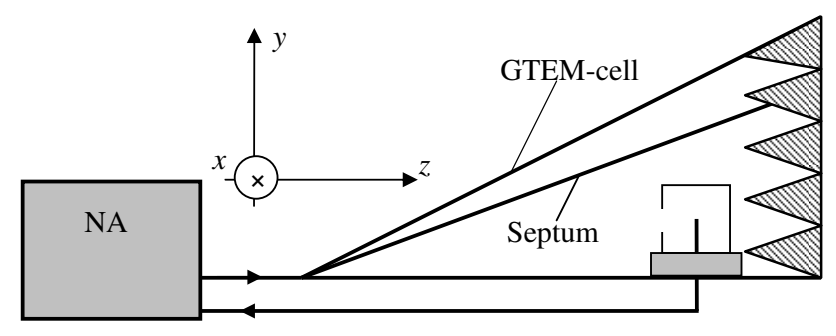

Fig. 3. Measurement setup inside the GTEM-cell.

This contribution is divided into 5 sections. After this introduction, the needed definitions for calculating the SE, TSE and EM-SE from electric and magnetic field measurements are given in Sect. 2. In Sect. 3, the numerical and the measurement results of the SE of the down-scaled shielding cabinet are presented. The measurement of the T-SE of four different shielding cabinets is shown in Sect. 4. In Sect. 5, concluding remarks are given.

\section{Definitions}

The transient shielding effectiveness (T-SE) introduced by Klinkenbusch (2005) is analytically derived from the examination of the ratio of electromagnetic energy absorbed by an unshielded load to that one absorbed by the shielded load for the same incident field at the limiting case that the load vanishes. It is based on the conventional measurement of the electric and magnetic SE at an arbitrary point $q$ inside an empty enclosure:

$\left.S E_{e}\right|_{q}=20 \log _{10} \frac{\left|\boldsymbol{E}^{\text {unshielded }}\right|_{q}}{\left|\boldsymbol{E}^{\text {shielded }}\right|_{q}} \mathrm{~dB}$

$\left.S E_{m}\right|_{q}=20 \log _{10} \frac{\left|\boldsymbol{H}^{\text {unshielded }}\right|_{q}}{\left|\boldsymbol{H}^{\text {shielded }}\right|_{q}} \mathrm{~dB}$.

The T-SE is defined by the expression

$$
\begin{aligned}
& \left.S E_{t}\right|_{q} \\
& =10 \log _{10} \frac{2 \int_{0}^{\infty}\left|S^{\text {inc }}(\omega)\right|^{2} \omega d \omega}{\int_{0}^{\infty}\left|S^{\text {inc }}(\omega)\right|^{2}\left[\frac{\left|\boldsymbol{E}^{\text {shielded }}\right|_{q}^{2}}{\left|\boldsymbol{E}^{\text {unshielded }}\right|_{q}^{2}}+\frac{\left|\boldsymbol{H}^{\text {shielded }}\right|_{q}^{2}}{\left|\boldsymbol{H}^{\text {unshielded }}\right|_{q}^{2}}\right] \omega d \omega} \mathrm{dB},
\end{aligned}
$$

where $S^{\text {inc }}(\omega)$ denotes the spectral density distribution of an arbitrary transient plane wave and $\omega$ denotes the angular frequency. It is a single value for a given or assumptive incident field pulse.

The frequency domain equivalent to the T-SE is the electromagnetic shielding effectiveness (EM-SE). This measure 
is derived from the examination of the ratio of electromagnetic power absorbed by an unshielded load to that one absorbed by the shielded load for the same incident field at the limiting case that the load vanishes. The EM-SE is defined by Eq. (1) as

$$
\left.S E_{\text {em }}\right|_{q}=10 \log _{10}\left[\frac{2}{\frac{\left|\boldsymbol{E}^{\text {shielded }}\right|_{q}^{2}}{\left|\boldsymbol{E}^{\text {unshielded }}\right|_{q}^{2}}+\frac{\left|\boldsymbol{H}^{\text {shielded }}\right|_{q}^{2}}{\left|\boldsymbol{H}^{\text {unshielded }}\right|_{q}^{2}}}\right] \mathrm{dB} .
$$

\section{Examination of the SE of a scaled model}

In order to examine the resonance phenomena inside the shielding cabinet, the SE of a down-scaled model (scaling factor 1:10) shall be analyzed numerically and practically. The advantage of such a small model is, that measurements can be performed inside a GTEM-cell in a laboratory.

\subsection{Building up a model}

Resonance phenomena can best be recognized, when no absorbing material is placed inside the enclosure. In addition, the aperture can be left open, so that more electromagnetic energy can couple into the enclosure. As a result, the rectangular shielding enclosure with rectangular aperture depicted in Fig. 2 is analyzed. The dimensions of the enclosure and the polarisation and orientation of the incident plane wave are also shown in Fig. 2. Due to the geometrical scaling factor, all resonance phenomena will occur at a ten times higher frequency, than they would occur in the shielding cabinet.

\subsection{Performing measurements and simulations}

For the numerical calculations, the MoM-tool CONCEPT II and the FEM-tool COMSOL MULTIPHYSICS were available. For the measurement of the SE, a metallic enclosure made of aluminum was built and placed inside a GTEM-cell 1250 as shown in Fig. 3. The GTEM-cell is connected to port 1 of a vector network analyzer (VNA). An electric monopole is placed vertically in the center of the enclosure and is connected to port 2 of the VNA. The electric SE is derived from the difference of the insertion loss $S_{21}$ without and with the enclosure in place in $\mathrm{dB}$. For the simulations, the amplitude of the incident plane wave is compared to the amplitude of the electric field in the center of the enclosure.

\subsection{Results}

Simulations and measurements were performed in the frequency range from $10 \mathrm{MHz}$ up to $2 \mathrm{GHz}$. The results are depicted in Fig. 4. The SE of the enclosure starts at about $20 \mathrm{~dB}$ and it drops down to about $-15 \mathrm{~dB}$ at approx. $700 \mathrm{MHz}$. Continuing to higher frequencies, the SE exhibits a maximum at approx. 1.1 GHz. Up to this frequency, the simula-

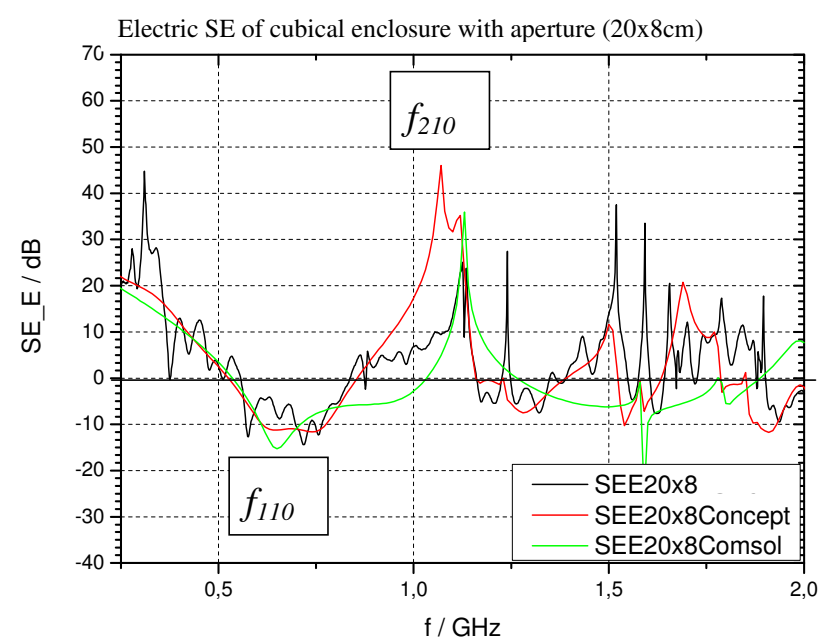

Fig. 4. Numerical calculation and measurement of the electric SE in the center of the enclosure.

tions and the measurement correlate well. For higher frequencies, the SE exhibits rapid changes from high to low values and vice versa. This behaviour of the $\mathrm{SE}$ is the characteristic behaviour of the enclosure and not of the shielding material. The enclosure is excited by its characteristic eigenfrequencies, which are resonating. The characteristic resonance frequencies can be calculated according to Zinke et al. (1986):

$f_{i j k}=\frac{1}{2 \sqrt{\mu \varepsilon}}\left(\sqrt{\left(\frac{\mathrm{i}}{\mathrm{a}}\right)^{2}+\left(\frac{j}{b}\right)^{2}-\left(\frac{k}{c}\right)^{2}}\right)$.

In this equation, $a, b$ and $c$ denote the three dimensions of the rectangular enclosure and $i, j$ and $k$ are integers greater or equal to zero with the restriction, that not more than one value of the three may be zero at the same time. According to Eq. (5), the first eigenresonance frequency $\left(f_{110}\right)$ for an enclosure of dimensions $a=b=c=0,3 \mathrm{~m}$ is approx. $700 \mathrm{MHz}$ at which the SE breaks down to negative values. The field distribution at this frequency inside the enclosure is shown in Fig. 5 upper left hand diagram. In the center of the enclosure, the field strength is higher than the incident field strength, thus causing the breakdown of the electric SE. The field distribution of the $f_{210}$ resonance frequency is shown in the upper right hand diagram of Fig. 5. From this diagram it can be recognized why the SE exhibits a maximum at this frequency: a field probe at the center of the enclosure can't detect a significant field strength because the field maxima are off center. Accordingly, the SE breaks down at the $f_{130}$ resonance frequency and it exhibits again a maximum at the $f_{230}$ resonance frequency. In general, the SE breaks down at all eigenfrequencies that have got an even field distribution along a line through the center of the enclosure, and it exhibits a local maximum at eigenfrequencies that have got an 

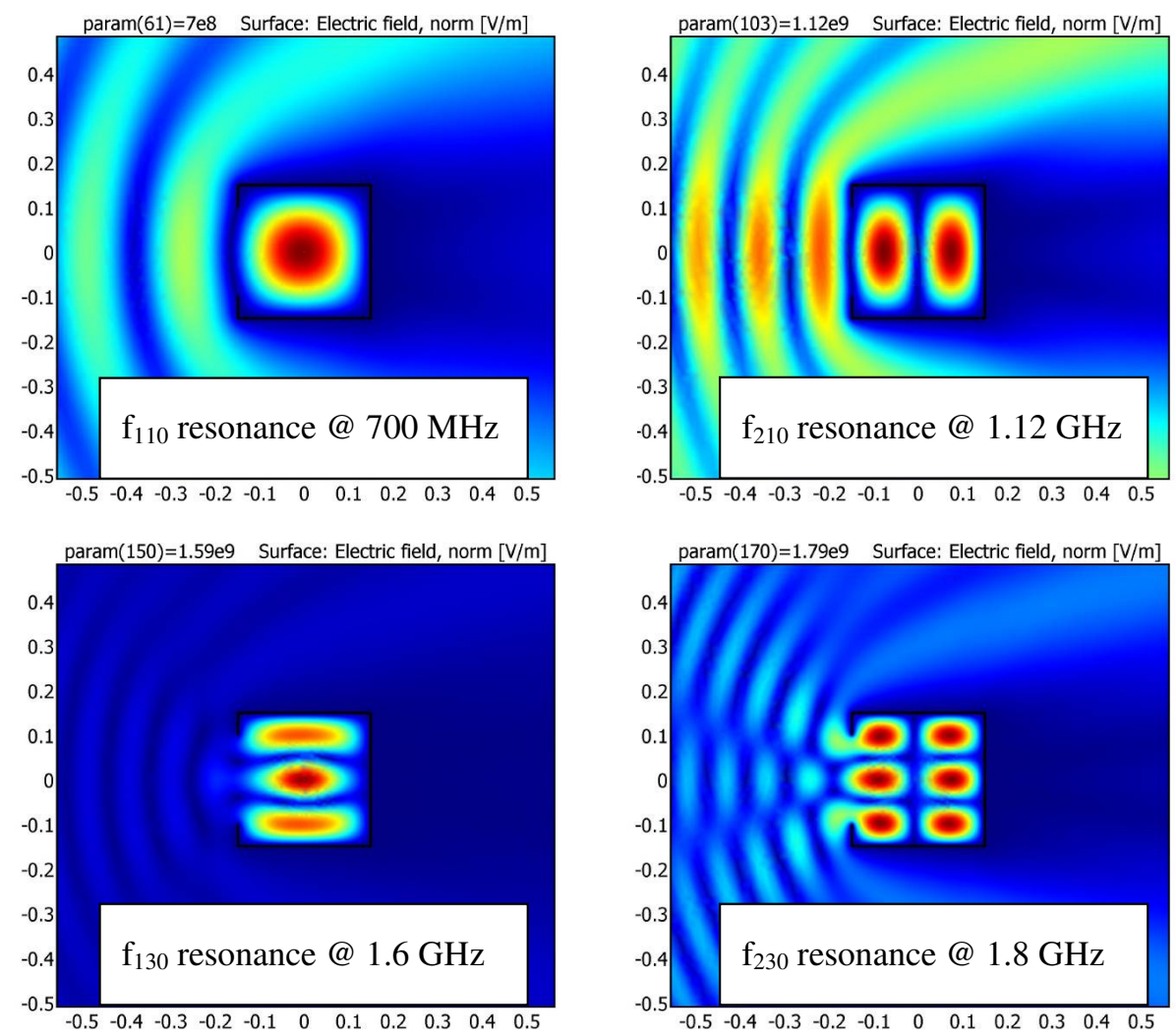

Fig. 5. Distribution of the electric field strength of some resonating modes inside the enclosure.

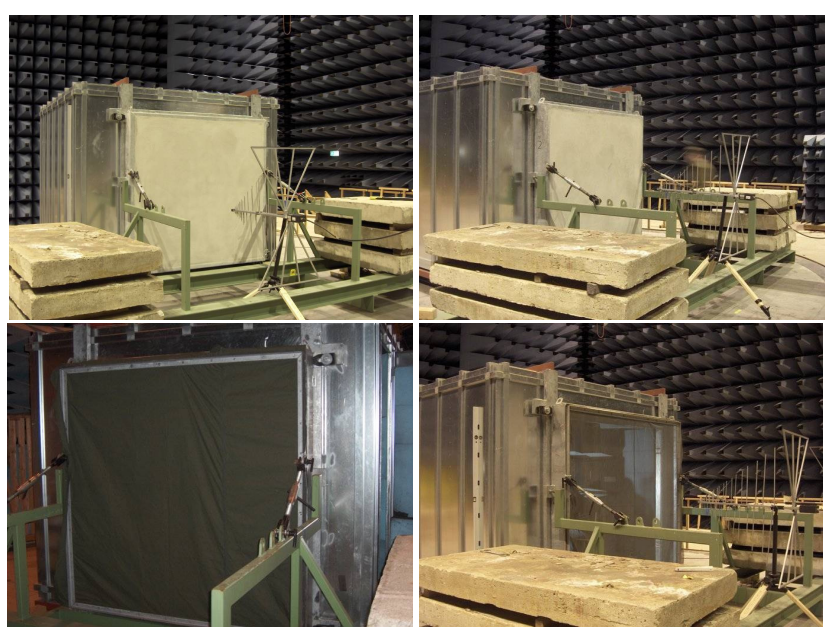

Fig. 6. Shielding cabinet with four different front walls. Top row: walls made of reinforced concrete. Bottom row: walls made of conductive textile.

odd field distribution, provided, the field measurements are performed in the enclosure's center.

\section{Measurement of the T-SE of shielding cabinets}

The transient shielding effectiveness (T-SE) is according to Eq. (3) composed of the measurement results of the electricand the magnetic SE of the empty shield and of the spectral density distribution of the field pulse under consideration. The T-SE therefore always denotes the protective property of the shield against a specific pulse. Klinkenbusch (1998) has suggested to use pulses with a Gaussian spectral density distribution for the examination of the T-SE of shielding enclosures. In doing so, the T-SE can be plotted as a function of the center frequency $f_{0}$ of such pulses for a given pulse bandwidth at half max (PBW50).

\subsection{Measurement setup}

The enclosure under test is a $3 \times 3 \times 2,5 \mathrm{~m}$ metallic cabin introduced by Frenzel (2007). In the inside, HF-foam absorbers of a thickness of approx. $10 \mathrm{~cm}$ are attached to the walls. One side wall contains a $1.9 \times 1.9 \mathrm{~m}$ squared opening, in front of which different shielding walls can be placed. The shielding walls contain a metallic frame which is thoroughly pressed against metallic gaskets, while the wall is mounted in front of the opening. By means of exchanging the shielding wall, a new measurement configuration is maintained. For the examinations in the course of this contribution, four dif- 

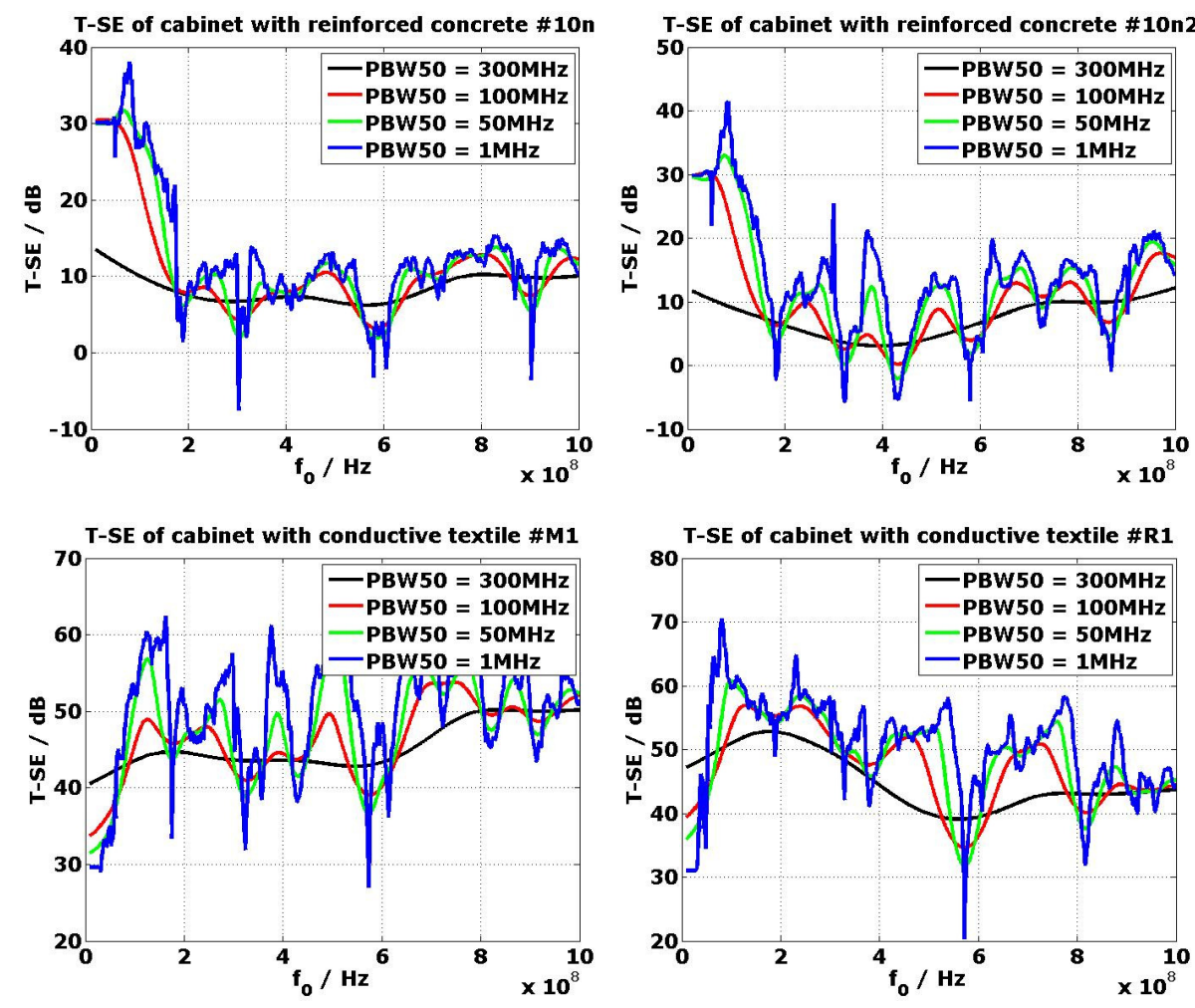

Fig. 7. Transient shielding effectiveness of the four measurement configurations of Fig. 6.

ferent shielding walls where available: two walls made of reinforced concrete and two walls made of conductive textile (see Fig. 6). The two concrete walls each have got two layers of a steel mesh. In the first wall (upper left hand picture of Fig. 6), the layers are welded together. The two textiles differ from each other in a way that the first textile (bottom left hand picture) contains woven metal filaments whereas the second textile (bottom right hand picture) is silver coated. The mesh of the reinforcement inside the concrete walls is about $7.5 \times 7.5 \mathrm{~cm}$ whereas the metallic mesh inside the textile has dimensions of only a few millimeters. Thus, it can be expected, that the shielding cabinets containing the conductive textile walls provide a better shielding than the cabinets containing the concrete walls.

The SE measurements are performed inside Europe's largest fully anechoic chamber (approx. $40 \times 16 \times 14 \mathrm{~m}$ ), which is operated by the Technical Center for Information Technology and Electronics / Electromagnetic Effects / EMC (WTD 81) of the German Armed Forces in Greding, south Germany. The measurement equipment for these tests is listed in Table 1. The bicon-log.-per.-antenna for the field generation is located outside the shielding cabinet in a distance of $2.2 \mathrm{~m}$ in front of the shielding wall. The electric and magnetic SE of the cabinet is derived from field measurements in the center of the cabinet with a dipole $(\mathrm{l}=15 \mathrm{~cm})$ and a loop antenna $(\mathrm{d}=8 \mathrm{~cm})$. For the measurement of the
Table 1. Measurement equipment for T-SE measurements.

\begin{tabular}{ll}
\hline & $\mathbf{3 0} \mathbf{M H z} \mathbf{- 1 ~ G H z}$ \\
\hline $\begin{array}{l}\text { Transmitter: } \\
\begin{array}{l}\text { Signal-generator } \\
\text { Antenna }\end{array}\end{array}$ & $\begin{array}{l}\text { Rohde \& Schwarz SMG 801.0001.52 } \\
\text { EMCO 3142C BiConiLog }\end{array}$ \\
\hline Amplifier & Ifi Model 5500, 10W \\
Receiver: & \\
Spectrum Analyzer & Rohde \& Schwarz FSP 30 \\
\hline Antennas & Dipole and loop-antenna \\
BALUN & ProDyn BIB-100G \\
\hline Pre-amplifier & Agilent 83006A \\
Cabling: & RG214 \\
\hline
\end{tabular}

incident fields, it was more feasible to remove the shielding wall only (i.e. leaving the $2 \times 2 \mathrm{~m}^{2}$ aperture open) instead of removing the whole shielding cabinet.

\subsection{Measurement results}

For the evaluation of the T-SE, Gaussian pulses with four different pulse bandwidths (PBW50) are used: PBW50$1=1 \mathrm{MHz}, \quad$ PBW50-2 $=50 \mathrm{MHz}$, PBW50-3=100 MHz and PBW50-4=300 MHz. The T-SE is evaluated for pulses 
with a center frequency ranging from $f_{0, \min }=30 \mathrm{MHz}$ up to $f_{0, \max }=1 \mathrm{GHz}$. The results for all four measurement configurations of the shielding cabinet are depicted in Fig. 7. The diagrams are arranged in accordance to the measurement setups in Fig. 6. From all four diagrams, it can be seen that examining the T-SE with narrowband pulses, the T-SE varies strongly as function of the center frequency $f_{0}$. This is not surprising: examining the T-SE with very narrow band pulses (e.g. PBW50=1 Hz) is equivalent to a frequency domain sweep. In fact, for the border case of a needle like spectrum of the "pulses", the T-SE becomes the EMSE. With rising pulse band width, the course of the T-SE becomes smooth. The examination with ultra wideband pulses (e.g. PBW50 $>300 \mathrm{MHz}$ ) is equivalent to penetrating the enclosure with dirac-like pulses.

Comparing the general course of the T-SE of the four measurement configurations for UWB pulses, the different shielding behaviour of the four cabinets can be recognized. The T-SE of the cabinets with concrete walls is in the range of $5-10 \mathrm{~dB}$, whereas the T-SE of the cabinets with conductive textile walls is in the range of $40-50 \mathrm{~dB}$. The oscillations of the T-SE as function of $f_{0}$ don't bear additional information. In general, it can be seen that examining the T-SE of enclosures with UWB pulses removes the strong ripples of the frequency domain SE measurements.

\section{Conclusions}

In the present work, the shielding behaviour of different shielding cabinets is analyzed. At first, numerical simulations are performed with CONCEPT II and COMSOL MULTIPHYSICS, in order to show the field distribution inside the enclosure at some of its eigenresonance frequencies. In case of determining the SE of the enclosure from measurements in its center, the SE breaks down at those eigenresonance frequencies which cause an even geometrical field distribution with field maxima in the measurement position. At eigenresonance frequencies that cause an odd geometrical field distribution, the SE exhibits local maxima because of a field knot of the standing wave pattern in the measurement position. These phenomena can be recognized in the course of the simulated SE as well as in the measured SE. For the measurements, a downscaled model of the shielding cabinets made of aluminum was built and placed inside a GTEM-cell.

The examination of the shielding cabinet's transient SE is done from SE measurements inside Europe's largest fully anechoic chamber. The examination is done with Gaussian pulses with center frequency $f_{0}$ and pulse bandwidth PBW50. The transient SE is analyzed as function of the center frequency of these pulses. In the border case of pulses with needle like spectra, the course of the T-SE is equivalent to the course of the electromagnetic SE. For ultra wideband pulses, the T-SE becomes a smooth curve. As a result, UWB pulses are best for analyzing the T-SE of enclosures. The practicability of the T-SE is currently investigated by the authors.

Acknowledgements. The authors like to thank the Technical Center for Information Technology and Electronics/Electromagnetic Effects/EMC (WTD81), being part of the German Armed Forces, for providing the needed measurement facilities.

\section{References}

CONCEPT II: Field calculation software based on the Method of Moments (MoM), the software was developed by the University of Technology, Hamburg-Harburg, Germany.

COMSOL Multiphysics: Field calculation software based on the Finite Element Method (FEM), the software was developed by COMSOL Multiphysics GmbH.

Frenzel, T., Stumpf, J., and Koch, M.: Shielding Effectiveness of Original and Modified Building Materials, Adv. Radio Sci. (Kleinheubacher Berichte 2006), Copernicus Gesellschaft e.V., Katlenburg-Lindau, 2007.

Herlemann, H., Koch, M., Camp, M., Bausen, A., and Sabath, F.: Measurement of Protection Properties of Closed Shields in Time Domain, EMC York 2004 International Conference and Exhibition, York, England, 2004.

IEEE Standard 299-1997: For Measuring the Effectiveness of Electromagnetic Shielding Enclosures, 1997.

Klinkenbusch, L.: Multipolanalyse des Schirmverhaltens bei transienter Ströraussendung, EMV 98, VDE-Verlag, Berlin, Offenbach, 1998.

Klinkerbusch, L.: On the Shielding Effectiveness of Enclosures, IEEE Transactions on EMC, 47, 3, 589-601, August 2005.

Marvin, A. C., Dawson, J. F., and Dawson, L.: New developments in shielding effectiveness measurements - shielding aperture, New EMC Issues in Design: Techniques, Tools and Components Seminar, The IEE , 45-50, 28 April 2004.

Marvin, A. C., Dawson J. F., Ward, S., Dawson, L., Clegg, J., and Weissenfeld, A.: A Proposed New Definition and Measurement of the Shielding Effect of Equipment Enclosures, IEEE Transactions on EMC, 47, 3, 589-601, August 2005.

Zinke, O., Brunswig, and Vlcek, A.: Lehrbuch der Hochfrequenztechnik, Erster Band, 3. Auflage, Springer-Verlag, Berlin Heidelberg, New York, Tokyo, 1986. 